Fig. 2 shows a specimen which was etched for a few minutes in 80 per cent alcohol. Etch pits became visible. On the left the surface is crowded with pits, on the right the pits are better resolved. As the pits are pointed they may be dislocations ${ }^{3}$. The left side seems to be the result of a crack passing slowly through the crystal. Such cracks are known to create a large number of dislocations at their edge.

If the crystals are heated to a high temperature $\left(600^{\circ}\right.$ C. $)$ and quenched, discontinuous lines become visible after etching. Fig. 3 shows a typical example on a dark field. It is suggested that the discontinuous lines consist of dislocations piled up against some obstacle.

If the surfaces are dissolved in water the number of observable slip sources is considerably reduced.

This work was carried out in the Institute for Experimental Physics of the University of Technology, Budapest. I wish to thank B. Jeszenszky for valuable discussions. A detailed paper will bo published in Acta Physica Hungarica.

Research Institute for Technical Physics,

\section{Z. MORLIN}

Hungarian Academy of Seiences, Budapest. Mar. 2

${ }^{1}$ Hollomon, J. H., and Fisher, J. C. (see Suzuki, T., "Dislocations and Mechanical Properties of Crystals (An International Conference)", 215
Londion, 1957).

' Jeszenszky, B., Acta Phys. Hung., 8, 147 (1957); Nature, 181, 499 (1958).

${ }^{3}$ Gilman, J. J., and Johnston, W. G., J. App. Phys., 27, 1018 (1956).

" Gilman, J. J., and Johnston, W. G., "Dislocations and Mechanical Properties of Crystals (An International Conference)", 116 (Wiley, New York, and Chapman and Hall, London, 1957).

\section{Unpleasantness of Distorted Sounds : a Criterion derived from the Distortion Spectrum}

For lack of any proved method of objective assessment the degree of non-linear distortion permissible in transmission systems which handle highquality speech and music programmes has been decided, up to the present, entirely on the basis of long experience. While collecting data to support a more factual approach to this subject the following experiments have been made.

Pulses of pure tone, rather like staccato piano notes, have been distorted in various ways and the 'unpleasantness' of the resulting sounds have been compared, and an objective criterion, $C_{t}$, computed. This shows satisfactory correlation with the unpleasantness and can be defined as follows:

$$
C_{t}=\sum_{2}^{n} n^{2}\left(p_{n}-t\right) ; \quad\left(p_{n}-t\right) \text { positive }
$$

where $n$ is harmonic number, $p_{n}$ is percentage amplitude of $n$th harmonic and $t$ that percentage of the signal (at its erest) which is just audible.

When the level of the crest of the pulse is 70 phons, and the sounds are presented under good listening conditions (for example, ambient noise in the region of 30 phons) a value of $t=0.2$ gives good correlation with subjective assessment. It is suggested that $t=0.5-1.0$ may be more appropriate so far as domestic broadcasting is concerned. Since $t$ is that harmonic percentage which is just audible, the value of $t$ will fall below these figures if the signal/noise ratio is increased, or rise if the ratio is reduced.

The validity of the criterion $C_{t}$ has been tested by setting up pairs of distortion spectra, which differ radically both in their general character and in their

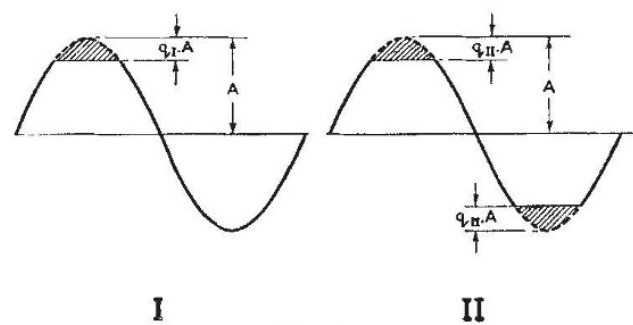

Fig. 1

band-width, and adjusting one of the spectra until the two sounds are equally unpleasant. If the signal frequency lies in the range $250-1,500 \mathrm{c} . / \mathrm{s}$., the value of the criterion $C_{t}$ derived from each spectrum will then be substantially the same, provided the appropriate value of $t$ is adopted; anomalies occur outside this frequency-range.

A form of distortion which is easily adjusted and eontrolled is shown at I in Fig. I; the proportion $q_{\mathrm{l}}$ of the amplitude, $A$, of the sine-wave can be varied by adjusting the steady bias applied to a diode shunting the signal path. The criterion $C_{t}$ increases roughly as $\sqrt{ } q$, when $t$ is made very small or zero. Distortion of the type shown at II in Fig. 1 is often found, although in a form very considerably attenuated by negative feedback, in high-power (for example, $150 \mathrm{~kW}$.) modulating-amplifiers for amplitudemodulated radio transmitters; in this case both positive- and negative-going parts of the wave are limited. The correlation between the criterion $C_{t}$ and the unpleasantness of both types of distortion has been investigated, and a report will be published in due course elsewhere.

The outcome of one set of experiments is as follows: it is found that when $q_{\mathrm{I}}=q_{\mathrm{II}}$, and $q$ is in the range $0.5>q>0$ the signal $\mathrm{I}$ in the figure is (within the limits of experimental error) as unpleasant as the signal II (although there is a great difference in tone-colour). On comparing the spectra the two criteria are found to differ by less than 10 per cent when $t$ is made zero and at least eight terms are summed.

Now when $q_{\mathrm{I}}=q_{\mathrm{II}}$ the spectrum of the signal II is similar to the spectrum of $\mathrm{I}$, except that all the odd terms are doubled in amplitude and the even terms are entirely absent. Thus on summing the terms to derive the criterion, the absence of half of them is made up for by the doubled amplitude of those remaining.

It is important to notice that roughly the same effect would have been obtained if $n$ or $n^{3}$ were substituted for $n^{2}$ in the equation; that the signals I and II sound equally unpleasant, therefore, provides no direct test of the criterion. However, it does demonstrate that any criterion (such as the total harmonic percentage) which is based upon the summation of terms in $\left(p_{n}\right)^{2}$, must necessarily fail to correlate with the unpleasantness.

There is some evidence that $\log \left(C_{t}\right)$ may be used to predict the 'rating' of the distortion; thus, if the rating-scale is expressed by a series of numbers such as: 1, 'distortion not perceptible'; 2, 'perceptible with effort'; 3 , 'perceptible without effort' and so on, the rating number tends to vary with $\log \left(C_{l}\right)$.

Research Department, E. R. WIGAN

British Broadcasting Corporation, Kingswood Warren,

Tadworth, Surrey. Feb. 16. 